\title{
Hacia el conectivismo: docente y estudiante, sus roles en el espacio virtual
}

\section{Connectivism: teacher and student, their roles in virtual space}

\section{Rumo ao conectivismo: professor e aluno, seus papéis no espaço virtual}

Carlos Alberto Vásquez Villanueva carlosv@upeu.edu.pe

https://orcid.org/0000-0002-0782-7364

Universidad Peruana Unión, Perú
Salomón Axel Vásquez Campos

salomonvasquez@upeu.edu.pe

iD https://orcid.org/0000-0001-9405-0794

Universidad Peruana Unión, Perú

Lizardo Vásquez Villanueva

lizar v@upeu.edu.pe

https://orcid.org/0000-0002-1516-1312

Universidad Peruana Unión, Perú

Recibido: 03 de enero de 2021

Aceptado: 06 de junio de 2021

\section{Resumen}

Las herramientas tecnológicas son de fácil acceso para unos; para otros no. La educación se orienta hacia el aprendizaje mediante diversos sistemas de internet. Cambia el rol del docente, también del estudiante. El presente artículo se respalda en objetivo: analizar el rol del docente y del estudiante, según el conectivismo. Con este afán, se abordan los conceptos: conectivismo y aprendizaje. Se exponen los fundamentos teóricos de la teoría conectivista. Se realiza el análisis del rol del docente y del estudiante según el conectivismo. Se concluye que el docente tiene el rol de guía, facilitador e impulsor de conexiones en redes de aprendizaje, el desarrollo de habilidades conectivas; el estudiante, por su parte, cumple el rol de aprendiz autónomo quien, con la guía del docente, contribuye a la red de aprendizaje colectivo y participa activamente en el flujo de conocimiento.

Palabras clave: conectivismo, rol del docente, rol del estudiante. 


\begin{abstract}
Technological tools are easily accessible for some; for others not. Education is oriented towards learning through various internet systems. Changes the role of the teacher, also the student. This article is supported by the objective: to analyze the role of the teacher and the student, according to connectivism. With this eagerness, the concepts are addressed: connectivism and learning. The theoretical foundations of the connectivist theory are exposed. The analysis of the role of the teacher and the student is carried out according to connectivism. It is concluded that the teacher has the role of guide, facilitator and promoter of connections in learning networks, development of connectivist skills; The student, for his part, fulfills the role of an autonomous learner who, with the teacher's guidance, contributes to the collective learning network and actively participates in the flow of knowledge.
\end{abstract}

Keywords: connectivism, teacher's role, student's role.

\title{
Resumo
}

As ferramentas tecnológicas são facilmente acessíveis para alguns; para outros não. A educação é orientada para a aprendizagem por meio de vários sistemas da Internet. Muda o papel do professor, também do aluno. Este artigo tem como objetivo: analisar o papel do professor e do aluno, segundo o conectivismo. Com esse desejo, são abordados os conceitos: conectivismo e aprendizagem. São expostos os fundamentos teóricos da teoria conectivista. A análise do papel do professor e do aluno é realizada de acordo com o conectivismo. Conclui-se que o professor tem o papel de guia, facilitador e promotor de conexões em redes de aprendizagem, no desenvolvimento de competências conectivas; O aluno, por sua vez, cumpre o papel de aprendiz autônomo que, com a orientação do professor, contribui para a rede coletiva de aprendizagem e participa ativamente do fluxo do conhecimento.

Palavras-chave: conectivismo, papel do professor, papel do aluno. 


\section{Introducción}

Los entornos virtuales del aprendizaje han empujado a todos sus actores hacia una nueva praxis, cuyos ejercicio docente se enfrenta a nuevos sistemas y modalidades, en todos los niveles, involucrando y describiendo los objetos y los sujetos totalmente heterogéneos, cuyas cargas administrativas y académicas son totalmente complejas, con plataformas proyectan discursos hegemónicos (Andrango, 2020). Actualmente, el conocimiento y la información se producen, se expanden y actualizan de un modo sorprendente, increíble, a una gran velocidad, jamás ha sido imaginada y observada por los docentes; los tiempos pasados han sido superados en todas sus formas, contenidos y metodologías. Todos los días se registran múltiples y nuevos descubrimientos y avances científicos. Las conexiones vía internet permiten tener acceso inmediato a ese sinfín de conocimiento producido en distintas partes del mundo, el cual genera un impacto grandioso sobre el aprendizaje. En este contexto, llega y se hace conocido el conectivismo, también sorprendente, valioso e inevitable para el aprendizaje de los estudiantes y para la información masiva de las poblaciones diversas, rompiendo espacios, tiempos y consignas pedagógicas formales; en este ámbito, tienen presencia notoria e inevitable las conexiones de fuentes de información (conocidas como nodos), cuya red permite el aprendizaje, gracias a la diversidad de conocimiento esparcido.

Para conectarse y responder a estas exigencias, la capacidad de actualizar el conocimiento propio comienza y se hace más útil, que conocer cierta información en un momento determinado.

En el ámbito y los entornos digitales, se percibe una desigualdad no solamente en los escenarios geográficos y viertuales, sino en los mismos sujetos; por ejemplo, la presencia de muchos profesores "migrantes digitales" y los estudiantes "nativos digitales" (Espinosa, 2017). Por su parte, Mattar (2018) destaca que el constructivismo y el conectivismo, en el ámbito de la tecnología, generan un "aprendizaje activo, situado, auténtico, experiencial y anclado".

Habiendo examinado los resultados favorables del conectivismo, y apuntando a su aplicación pedagógica, se encontró la necesidad de recopilar información científica con el objetivo de realizar un análisis del rol del docente y del estudiante según el conectivismo. Por su parte, Polo (2020) destaca que el conectivismo obliga a los docentes resolver los diversos problemas relacionados con los aprendizajes significativos y el rendimiento académico, para lo cual cada docente debe agenciarse de un método pertinente, orientado a la resolución de los problemas, alimentando los diálogos y las 
prácticas entre docentes y estudiantes. Basurto, Moreira, Velásquez y Rodríguez (2021) sustentan que el conectivismo, más allá de una teoría innovadora, mejora el proceso de enseñanza aprendizaje, superando los modelos tradicionales, los cambios sociales, los sistemas de la era digital. Prado (2021) se ocupa del conectivismo en el sentido de que las instituciones educativas deben trabajar sus procesos de enseñanza aprendizaje conectados a los objetivos de la sociedad globalizada.

Para el desarrollo del presente artículo, en primer lugar, se recopilan conceptos de conectivismo y aprendizaje. En segundo lugar, se exponen los fundamentos teóricos de la teoría conectivista. Por último, se aborda el tema objetivo de este artículo: el análisis del rol del docente y del estudiante según el conectivismo.

\section{Conectivismo}

\section{Concepto}

El conectivismo, llamado también aprendizaje distribuido, es "una nueva filosofía de la educación para la era digital”, la cual amplía y flexibiliza "el concepto de Zona de Desarrollo Próximo (ZDP)”, cuyo concepto le pertenece a Vygotsky, cuyo aprendizaje "se encuentra fuera del alumno, en redes sociales y herramientas tecnológicas"; es "una nueva e importante teoría, que incluye su visión pedagógica y práctica en cursos masivos y abiertos en línea" (Mattar, 2018, p. 202).

Para Teixeira y Martini (2019, p. 1015), “O conectivismo segundo abordagem de George Siemens (2004) é uma nova teoria da aprendizagem que reconhece que mudanças na sociedade da era digital são fatores determinantes para alterações nos ambientes instrucionais". Se destaca que el conectivismo es una teoría de aprendizaje, cuya teoría explica y sustenta los cambios generados en la sociedad digital. Para postular esta teoría, George Siemens investigó y observó las limitaciones del conductismo, constructivismo y cognitivismo, asimismo se basó sobre "las teorías de caos, redes, complejidad y autoorganización" (Siemens, 2007), las cuales fueron integradas para dar vida a los conceptos del conectivismo.

De acuerdo con Vallejo, Zambrano, Vallejo y Bravo (2019), las tendencias educativas apuntan a entender el conectivismo como "un constructo teórico complejo centrado en conexiones especializadas orientadas a encontrar, realizar, cultivar y mantener relaciones entre diferentes ideas, conceptos y campos del saber" (p. 526). Es la integración de principios, los cuales han sido generados por la teoría de caos, redes, complejidad y auto organización (García y Peña, 2019). 
Para Cueva, García y Martínez (2020), el conectivismo, en relación las TICs, es un enfoque pedagógico, es un modelo, es "una teoría para enriquecer el proceso de enseñanza aprendizaje", presenta complejidades en los procesos, durante el uso de las TICs, la búsqueda, el procesamiento y la comunicación de la información. Basurto et al., 2021) sustentan que el conectivismo es una teoría innovadora, comprometida con la fortaleza del proceso enseñaza aprendizaje. Es "una teoría de aprendizaje de la nueva era digital, aborda estrategias de aprendizaje que cubran las expectativas actuales de los estudiantes como herramienta innovadora en el proceso enseñanza-aprendizaje” (p. 235). También explica "cómo las tecnologías y el internet han creado grandes oportunidades", haciendo que los aprendizajes de los estudiantes sean rápidos y mejores, en un contexto donde se observa la información compartida en línea, la construcción de conexiones y la creación del conocimiento integrado.

\section{Aprendizaje y conectivismo}

En esta nueva concepción, el aprendizaje no ocurre únicamente dentro del individuo, sino que sucede en una red de relaciones conectadas (Teixeira y Martini, 2019). El proceso enseñanza aprendizaje mejora mediante teorías innovadoras; por ejemplo, el conectivismo, cuyo proceso es continuo, perenne, evolutivo, mediante el uso de nuevas tecnología, con mucho esfuerzo y uso de tiempo, en un entorno abierto para los docentes y los estudiantes (Basurto et al., 2021). El aprendizaje responde a los nuevos objetivos de la sociedad globalizada (Prado, 2021).

\section{La teoría del aprendizaje para la era digital}

A la luz de los estudios de Siemens (2007), el conectivismo es dirigido por la premisa de que la toma de decisiones está basada en principios que sufren cambios continuos. Constantemente se está actualizando y adquiriendo información nueva. Para explicar el conectivismo, Siemens (2007) aclara que el individuo es el punto inicial de la teoría. El conocimiento personal está compuesto por una red que contribuye, con su conocimiento, a instituciones y organizaciones. Estas entidades, a su vez, retroalimentan a las redes, de esa manera proveen nuevo aprendizaje para los que formen parte de la red. Explicado de forma simplificada, en el conectivismo, el individuo influye y retroalimenta a las redes, así como las redes influyen y retroalimentan al individuo. El ciclo de flujo y desarrollo de conocimiento es el siguiente: "conocimiento personal a la red, de la red a la institución”, lo cual permite que los aprendices tengan el conocimiento más actualizado dentro de su área, gracias a las conexiones establecidas. 
Cabe recalcar que, dentro de la concepción de Siemens, la red puede ser definida simplemente como conexiones entre entidades. Las redes eléctricas, redes de computadoras y redes sociales funcionan con base en los mismos principios simples: Las personas, los grupos, los sistemas, los nodos y las entidades pueden conectarse para crear un todo integrado. Vallejo et al. (2019) refieren que la red es un espacio democrático, donde se distinguen diversos puntos de vista, los cuales aseguran la diversidad de información en el proceso de aprendizaje. Además, la red permite a los usuarios determinar y colaborar con los individuos que ellos quieren conocer, e identificar las materias en que ambos tienen interés común (Anderson y Dron, citados por Vallejo et al., 2019).

Cuando de aprendizaje en red se habla, las Tecnologías de la Información y Comunicación (TIC) son usadas para facilitar conexiones entre entidades de una comunidad de aprendizaje con sus respectivos recursos. La palabra clave en este contexto es "conexiones". El carácter relacional es el que se necesita, ya que el aprendizaje ocurre mediante las relaciones con los demás, así como con los recursos de aprendizaje. Dentro del conectivismo, el aprendizaje sucede en red y se genera en el contexto de una estructura combinada de vínculos sociales (red de aprendizaje). Lo que distingue a una red de aprendizaje es que está orientada a la generación colaborativa de conocimiento. La red está compuesta por personas con un interés específico y colectivo, siendo ese interés el principal motor para la interacción entre las personas que desean engrandecer la experiencia de aprendizaje en entornos educativos formales (instituciones y organizaciones) e informales: redes de colaboración espontánea y consultas (Solórzano y García, 2016).

Principios, recursos y componentes

Entre diversos principios, el conocimiento conectivo es individual y colectivo, generado mediante las conexiones y las redes; este conocimiento se cultiva, genera una experiencia propia, se constituye en la base del aprendizaje (García y Peña, 2019). Para Solórzano y García (2016, citados por García y Peña, 2019), existen otros principios: el conocimiento conectivo es el resultado de conexiones y acciones, revela la capacidad de distinción de lo que es importante, el carácter social de aprendizaje, el carácter activo y dinámico del estudiante, la integración y cohesión del grupo, la "relación actividadtecnología digital-conexiones".

El conectivismo tiene sus propios recursos; por ejemplo, los recursos educativos abiertos, los libros de textos digitales, los materiales digitales abiertos, (García \& Peña, 
2019). Los componentes del conocimiento conectivo son: motivación, interés, atención, adquisición, comprensión e interiorización, asimilación, aplicación, transferencia, evaluación (García y Peña, 2019).

Importancia

El conectivismo es muy importante para los aprendizajes de los estudiantes en espacios virtuales; por ejemplo, permite el encuentro, conocimiento, conectividad, aceptación y convivencia académica de los profesores "migrantes digitales" con los estudiantes "nativos digitales" (Espinosa, 2017). Genera la presencia del "aprendizaje activo, situado, auténtico, experiencial y anclado", en la experiencia de los estudiantes (Peña, 2020).

Reclama la implementación de recursos básicos para la conexión y el desarrollo de los procesos de aprendizaje (García y Peña, 2019). En el estudiante permite la generación de una mente extendida, para compartir los aprendizajes en espacios y tiempos virtuales (Sánchez, Peñalosa, Cárdenas y Villalobos, 2019). Por otro lado, facilita el desarrollo del rol de estudiante, en el marco de ámbito de compromiso académico muy sustantivo (Maluenda, Moraga y Díaz, 2019); condiciona el uso de las TICs para el desarrollo del proceso enseñanza aprendizaje (Cueva et al., 2020); permite el desarrollo profesional del docente y la educación a distancia y virtual (Peña, 2020); fortalece la relación profesorestudiante en la época de cuarentena, superando los desafíos y limitaciones, aprovechado las oportunidad de aprendizaje para los estudiantes en los entornos virtuales (Soto, 2020). Permite el encuentro entre docentes y estudiantes, en el ámbito de enfoques singulares, propios de espacios virtuales (Valderrama, 2020). Genera las posibilidades educativas, realizadas en realidades virtuales y combinadas (Oliveros y Núñez, 2020). Permite aprovechar su propia consolidación, en la condición de un enfoque pedagógico, para mejorar el desarrollo del proceso de aprendizaje virtual (Bernal, 2020).

Conectivismo y roles de los actores

\section{Rol del docente}

La mayor parte de los docentes y los espacios virtuales ya han sido familiares, por varias décadas; ambos siempre han caminado de la mano, para ampliar y fortalecer los procesos de enseñanza aprendizaje, aplicando los principios y las metodologías propuestas por el conectivismo; sin embargo, el año 2020, por el COVID-19, se fortalecieron mutuamente; además de experimentar variantes inesperadas, generando nuevas experiencias y modificaciones de los espacios, los tiempos, los sistemas y las plataformas virtuales. 
Anteriormente, el acceso al conocimiento era limitado y la única fuente de información verdadera e infalible era el docente. Sin embargo, el desarrollo del conocimiento humano pone fin a esta limitación, porque se construyen los espacios virtuales, quedando enlazados los docentes y los estudiantes. En dicho marco contextual y virtual, el rol del docente tiene múltiples manifestaciones: seleccionar, analizar e interpretar la información existente en internet (Espinosa, 2017).

El docente no es policía ni controlador. Verdaderamente, estas limitaciones fueron superadas largamente, gracias a la llegada y el desarrollo del internet, rompiendo las limitaciones de espacios y tiempos. Actualmente, se posee una vasta información, la cual es reconocida por los profesionales y no los profesionales, cuyo alcance y dominio depende solamente de un clic. En este contexto, muchos jóvenes, adolescentes y niños son actualmente catalogados "nativos internautas" y muy hábiles en el uso de herramientas tecnológicas; sin embargo, estas aptitudes no siempre garantizan que sepan cómo usarlas para su aprendizaje, su desarrollo cognitivo y humano. En este sentido, el docente se desempeña el rol de "organizador, guía, generador, acompañante, coacher, gestor del aprendizaje, orientador, facilitador, tutor, dinamizador o asesor" (Viñals y Cuenca, 2016, p. 110)

No solamente es un universo de espacios, tiempos, materiales y recursos económicos. También significa el encuentro de personas sensibles y sus particulares psicológicas. No significa la ausencia de estas particularidades humanas y sensible. En este espacio virtual, no se debe perder de vista la empatía entre el docente y el estudiante; en realidad, "en el dominio afectivo, la empatía implica la capacidad de unirse a las experiencias y sentimientos de la otra persona" (Valderrama, 2020, p. 66). De acuerdo con Prado (2021), el conectivismo sostiene que el docente no solamente entrega "una guía de lecturas, conceptos, materiales didácticos mediante herramientas digitales $\mathrm{y}$ multimedia, entre otros", "no tiene el control total sobre el proceso de enseñanzaaprendizaje" (p. 11).

\section{Rol del estudiante}

Además, los estudiantes participarán en la toma de decisiones, en respuesta a su propia motivación y su sentido de pertenencia. Esta orientación reconoce que los estudiantes se involucran activamente en la construcción de importantes predicciones relacionadas con la identidad y su futuro, sin descuidar el "fuerte rol de asistencia y dirección educativa" (Maluenda et al., 2019). Así como al docente se reclama mucha sensibilidad humana, también al estudiante se le mide y se valora por la misma: la 
sensibilidad humana. Es decir, en el ambiente donde ambos participan deben existir marcas de respeto permanente, colaboración, comunicación, respuestas, en forma recíproca, de ida y vuelta. Se necesita también la empatía del estudiante, es muy importante y alentadora (Valderrama, 2020). Los estudiantes necesitan poseer una mente extendida, cuya tesis considera "la cognición humana como un proceso que ocurre de manera compartida", en cuyos entornos los estudiantes muestran sus capacidades individuales para construir conexiones y redes de aprendizaje, en espacios y "entornos educativos no formales e informales en internet" (Sánchez et al., 2019). Según Prado (2021), en el contexto de la educación, el aprendizaje, proceso enseñanza aprendizaje y el concetivismo, el estudiante "tiene la oportunidad de elegir su proceso, mediante la construcción de sus propios recursos, involucrándose activamente y pre-enseñándose, de esta manera demuestra su comprensión y aprendizaje" (p. 11).

\section{El conectivismo durante la COVID-19}

El conectivismo ha permitido el cambio de las prácticas pedagógicas, las visiones, la formación, el desarrollo profesional, permitiendo el uso de las redes sociales, las diversas plataformas, el uso de teléfonos celulares, tablets, la modalidad e-learning, la unión de países, la formulación de proyectos múltiples (Peña, 2020). El COVID-19, sorpresivo para todo el mundo, significó la presencia de muchas renuncias a ciertos paradigmas tradicionales y la creación y la asunción de nuevos, los cuales han sido ensayados, reforzados, condicionados, reconstruirlos y adaptarlos a la diversidad de los espacios, las condiciones económicas, a los niveles de enseñanza, a las nacionalidades, a las culturas de los participantes, a las políticas de los estados.

La COVID-19, en el mundo y en el Perú, obligó que los países y sus respectivos ministerios de educación realizaran cambios radicales en los espacios educativos de todos los niveles, dispusieran su respectiva plataforma virtual, involucrando a los docentes, los estudiantes, los padres de familia y las mismas autoridades del sector, generando desafíos, oportunidades e interrelaciones mediante el conectivismo; es decir, mediante "distintas plataformas de carácter e-learning para monitorear y cautelar la debida progresión curricular" (Soto, 2020). Estas plataformas fueron construidas y ofrecidas, con mucha desesperación, en forma ambiciosa, con demasiada "autoridad", con las exigencias sin cálculos previstos, solamente con las buenas intenciones de responder a las exigencias de las autoridades superiores y la realización de los procesos de enseñanza aprendizaje, en 
cuyos procesos los protagonistas (docentes, estudiantes y padres de familia) se matricularán sin experiencia, sin visión, sin intuición, con mucho miedo por el provenir. Además, durante el COVID-19, se pretende estudiar cómo se desarrollan las relaciones y las interrrelaciones de dos sujetos: estudiantes y docentes, cuyos protagonismos son desarrollados en espacios virtuales y mediante el conectivismo; en este sentido, se propone reflexionar en torno a las nuevas perspectivas que reinterpretan la importancia de la relación docente-aprendiz en el proceso de aprendizaje recíproco que se produce entre ambos agentes, así como evaluar los alcances y oportunidades que ofrece la educación virtual en el contexto globalizado contemporáneo (Soto-córdova, 2020, p. 75).

Por otro lado, estos espacios virtuales de aprendizaje serán significativos, si se investigan las "diversas interacciones entre docente y aprendiz", "la función social y el modelo pedagógico de las instituciones educativas", "las competencias que han de tener los/as docentes", "los efectos de la empatía y su importancia" (Valderrama, 2020). El conectivismo, teoría emergente de aprendizaje, permite el desarrollo de "la alfabetización en información y la visual literacy, como conocimientos especializados de la bibliotecología que tratan sobre el uso y reconocimiento de la información" (Oliveros y Núñez, 2020, p. 46). Bernal (2020) aprecian que el conectivismo constituye un enfoque pedagógico, para lograr el desarrollo de los procesos de aprendizaje, "desde las tecnologías de la información y la comunicación aplicadas a la educación”.

\section{Conclusiones}

La educación implica la movilización de los sujetos en un escenario complejo, mucho más cuando las circunstancia, las respuestas, los recursos, las evaluaciones, las metodologías, los procesos no han sido planificados previamente y con la inteligencia pertinente. En el contexto de este estudio, se subrayan, implícita y explícitamente, varios conceptos inevitables, de los cuales no se prescinde cognitivamente, aunque no hayan sido referidos y abordados en su totalidad: enseñanza, aprendizaje, docentes, estudiantes, virtualidad, conectivismo, entre otros.

En el escenario conectivista, el docente, en la condición de novato y/o experto, desempeña el rol de guía, facilitador e impulsor de conexiones en las diversas redes de aprendizaje, sembrando aceptación, motivación, aliento, ánimo, conexión, diálogo, satisfacción, empatía, tolerancia, comunicación, en los estudiantes y en los mismos padres de familia, quienes sostienen y, además, fortalecen los escenarios del proceso enseñanza aprendizaje y las plataformas virtuales. 
Una de las enseñanzas más importantes que los docentes dejan a sus estudiantes es la capacidad de filtrar y seleccionar información actualizada, relevante y confiable, así como saber analizar e interpretar dicha información. Los docentes se enfocan en enseñar y cultivar las habilidades conectivistas de los estudiantes; es decir, habilidades que hagan posible los diversos aprendizajes virtuales y el establecimiento de conexiones enriquecedoras, respondiendo, a la vez, a las exigencias de la sociedad globalizada. No solamente realicen la entrega los materiales y los recursos para el desarrollo del proceso enseñanza aprendizaje, sino controlen, trasformen, enriquezcan dicho proceso.

Por su lado, el estudiante también es un poseedor de conocimiento. Cumple el rol de aprendiz autónomo que, con la guía del docente, contribuye para enriquecer la red del aprendizaje colectivo y participa activamente para generar el flujo de conocimiento; en otros términos, el estudiante influye sobre los demás homólogos y retroalimenta las redes. El estudiante, en este entorno, asume una postura activa, dinámica, crítica; aprende por sí mismo, con las requeridas responsabilidad y autonomía; en los entornos virtuales, usa sus capacidades, destrezas, habilidades, madurez y desarrollo psicológico. También el estudiante ejerce auto disciplina, auto aprendizaje, trabajo colaborativo, gestión a sí mismo, generación de conocimiento, interacción, contribución, respeto a las normas éticas, generación de conciencia, toma de decisiones, motivación propia, sentido de pertenencia, sensibilidad humana, respeto permanente, colaboración, respuestas, empatía, mente extendida, construcción de sus propios recursos.

\section{Referencias}

Andrango, L. P. (2020). Entorno virtual de aprendizaje de estudios sociales para estudiantes de los novenos años de educación básica general. [Tesis de magíster, Universidad Tecnológica Israel]. Recuperado de http://repositorio.uisrael.edu.ec/bitstream/47000/2578/1/UISRAEL-EC-MASTEREDUC-378.242-2020-070.pdf

Basurto, S. T., Moreira, J. A., Velásquez, A. N. y Rodríguez, M. (2021). El conectivismo como teoría innovadora en el proceso de enseñanza-aprendizaje del idioma inglés. Pol. Con., 6(1), 234-252. https://doi.org/10.23857/pc.v6i1.2134

Bernal, E. (2020). Aportes a la consolidación del conectivismo como enfoque pedagógico para el desarrollo de procesos de aprendizaje. Revista Innova Educación, 2(3), 394-412. https://doi.org/10.35622/j.rie.2020.03.002 
Cueva, J. L., García, A. y Martínez, O. A. (2020). La influencia del conectivismo para el uso de las TIC en el proceso enseñanza-aprendizaje. Revista Dilemas Contemporáneos: Educación, Política Y Valores, VII(2), 1-28. Recuperado de http://www.dilemascontemporaneoseducacionpoliticayvalores.com

Espinosa, A. D. (2017). Profesores "migrantes digitales" enseñando a estudiantes “nativos digitales." Medisur, 15(4), 463-473. Recuperado de https://www.medigraphic.com/pdfs/medisur/msu-2017/msu174d.pdf

García, S. y Peña, S. (2019). Conectivismo y proceso de aprendizaje de las ciencias sociales en estudiantes de educación general básica superior. Implementación de recursos educativos basados en el conectivismo. [Tesis, Universidad de Guayaquil]. Recuperado de http://repositorio.ug.edu.ec/bitstream/redug/46108/1/BFILO-PSM-19P154.pdf

Gutiérrez, L. (2012). Conectivismo como teoría de aprendizaje: conceptos, ideas, y posibles limitaciones. Revista Educación Y Tecnología, (1), 111-122. Recuperado de https://dialnet.unirioja.es/servlet/articulo? codigo $=4169414$

Maluenda, J. I., Moraga, F. y Díaz, A. (2019). El rol del estudiante en el fenómeno del compromiso académico en educación superior. Wimblu, Rev. Estud. de Psic. y Cienc. Soc., 14(1), 81-94. Recuperado de https://www.medigraphic.com/pdfs/medisur/msu-2017/msu174d.pdf

Mattar, J. (2018). El constructivismo y el conectivismo en tecnología educativa: El aprendizaje activo, situado, auténtico, experiencial y anclado. RIED. Revista Iberoamericana de Educación a Distancia, 21(2), 201-217. http://dx.doi.org/10.5944/ried.21.2.20055

Oliveros, S. y Núñez, C. (2020). Posibilidades educativas de la realidad virtual y la realidad combinada: una mirada desde el conectivismo y la bibliotecología. Revista Saberes Educativos, (5), 46-62. Recuperado de https://revistateoriadelarte.uchile.cl/index.php/RSED/article/view/57783/63384

Peña, M. (2020). Desarrollo profesional docente y educación a distancia. Una experiencia desde la cooperación sur-sur. Revista Saberes Educativos, (5), 100119. https://doi.org/10.5354/2452-5014.2020.57818 
Polo, L. E. (2020). La resolución de problemas: una mirada desde el aprendizaje significativo y el conectivismo. Acta Herediana, 63(1), 55-60. https://doi.org/10.20453/ah.v63i1.3702

Prado, A. B. (2021). Conectivismo y diseño instruccional: ecología de aprendizajes para la universidad del siglo XXI en México. Revista de Educación de la Universidad de Málaga, 2(1), 4-20. https://doi.org/10.24310/mgnmar.v2i1.9349

Quintana, E., Vidal, D., Torres, L., Castrillejo, V. A., Santamaría, F., Alonso, N. (Traductores). (2010). George Siemens Conociendo el conocimiento. [Grupo Nodos Ele]. Recuperado de http://davidal.es/wpcontent/uploads/2020/09/Siemens.Conociendoelconocimiento.pdf

Rugeles, P. A., Mora, B. y Metaute, P. M. (2015). El rol del estudiante en los ambientes educativos mediados por las TIC. Revista Lasallista de Investigacion, 12(2), 132138. Recuperado de https://www.redalyc.org/pdf/695/69542291025.pdf

Said, E. (Ed.). (2013). Cooperación, comunicación y sociedad: escenarios europeos y latinoamericanos. Barranquilla, Colombia: Editorial Universidad del Norte. https://doi.org/10.13140/RG.2.1.2288.5606

Sánchez, J. M., Peñalosa, E. A., Cárdenas, M. G. y Villalobos, F. M. (2019). Análisis de redes y cognición en ambientes conectivistas de aprendizaje con inteligencia artificial. Revista Digital Internacional de Psicología y Ciencia Social, 5(1), 3758. https://doi.org/10.22402/j.rdipycs.unam.5.1.2019.176.37-59

Solórzano, F. y García, A. (2016). Fundamentos del aprendizaje en red desde el conectivismo y la teoría de la actividad. Revista Cubana de Educación Superior, 53(3), 98-112. Recuperado de http://scielo.sld.cu/pdf/rces/v35n3/rces08316.pdf

Soto, I. (2020). La relación estudiante-docente en tiempos de cuarentena: desafíos y oportunidades del aprendizaje en entornos virtuales. Revista Saberes Educativos, (5), 70-99. https://doi.org/10.5354/2452-5014.2020.57816

Teixeira, D. y Martini, S. C. (2019). Conectivismo Pedagógico: novas formas de ensinar e aprender no século XXI. Revista Thema, 16(4), 1012-1025.

http://periodicosnovo.ifsul.edu.br/index.php/thema/article/view/1583 
Valderrama, N. (2020). Docente y aprendiz: enfoques en espacios virtuales. Revista Saberes Educativos, (5), 63-69. https://doi.org/10.5354/2452-5014.2020.57815

Vallejo, P. A., Zambrano, G., Vallejo, P. Y. y Bravo, G. M. (2019). Importancia del Conectivismo en la inclusión para mejorar la Calidad Educativa ante la tecnología moderna. Revista Arbitrada Interdisciplinaria KOINONIA, IV(8), 522-543. http://dx.doi.org/10.35381/r.k.v4i8.297

Viñals, A. y Cuenca, J. (2016). El rol del docente en la era digital. Revista Interuniversitaria de Formación del Profesorado, 86(30.2), 103-114. Recuparado de https://dialnet.unirioja.es/servlet/articulo? codigo $=5670199$ 